\title{
Rapid Determination of Isomeric Benzoylpaeoniflorin and Benzoylalbiflorin in Rat Plasma by LC-MS/MS Method
}

\author{
Chuanqi Zhou ${ }^{1,2}$ and Xiaoke Wang ${ }^{1,2}$ \\ ${ }^{1}$ Key Laboratory of Molecular Diagnosis and Medicinal Chemistry, Ministry of Education, Hebei University, Baoding 071002, China \\ ${ }^{2}$ College of Chemistry \& Environmental Science, Hebei University, Baoding 071002, China \\ Correspondence should be addressed to Chuanqi Zhou; chuanqizhou@163.com
}

Received 15 January 2017; Revised 17 February 2017; Accepted 21 February 2017; Published 8 May 2017

Academic Editor: Frantisek Foret

Copyright (c) 2017 Chuanqi Zhou and Xiaoke Wang. This is an open access article distributed under the Creative Commons Attribution License, which permits unrestricted use, distribution, and reproduction in any medium, provided the original work is properly cited.

\begin{abstract}
Benzoylpaeoniflorin (BP) is a potential therapeutic agent against oxidative stress related Alzheimer's disease. In this study, a more rapid, selective, and sensitive liquid chromatography-tandem mass spectrometric (LC-MS/MS) method was developed to determine BP in rat plasma distinguishing with a monoterpene isomer, benzoylalbiflorin (BA). The method showed a linear response from 1 to $1000 \mathrm{ng} / \mathrm{mL}(r>0.9950)$. The precision of the interday and intraday ranged from 2.03 to $12.48 \%$ and the accuracy values ranged from -8.00 to $10.33 \%$. Each running of the method could be finished in 4 minutes. The LC-MS/MS method was validated for specificity, linearity, precision, accuracy, recovery, and stability and was found to be acceptable for bioanalytical application. Finally, this fully validated method was successfully applied to a pharmacokinetic study in rats following oral administration.
\end{abstract}

\section{Introduction}

Alzheimer's disease $(\mathrm{AD})$ is a chronic neurodegenerative disease that accounts for $60 \%$ to $70 \%$ of cases of dementia $[1,2]$. Oxidative stress has been generally recognized as a cause of various neurodegenerative disorders including $\mathrm{AD}$ $[3,4]$. Therefore, diverse antioxidants have been investigated as potential therapeutic agents against oxidative stress related AD. Benzoylpaeoniflorin (BP), a kind of monoterpene glycoside mainly isolated from Paeonia species, has been proved on strong antioxidant property in primary cultures of rat cortical cells against $\mathrm{H}_{2} \mathrm{O}_{2}$-induced neurotoxicity [5]. Recent attention has been paid to a role of $\mathrm{BP}$ in individual animal because exciting progress in the field of neurotoxicity is emerging through the establishment of experimental implementation of antioxidant activity. However, the quantification of BP in plasma and its role in neurotoxicity is yet to be identified. The goal of our analytical study is to develop a simple, sensitive, accurate, and reproducible analytical method for BP quantification in rat plasma, available for use in the dynamic analysis of the BP concentration profile in animal.
Several kinds of methods have been proposed for the determination of BP, based on HPLC with UV detection or LC-MS/MS [6-9]. In particular, the LC-MS/MS method is expected to be a powerful tool for the quantification of BP in biological fluids, because it generally offers superior selectivity and sensitivity for the detection of low concentration analytes in a complex matrix, such as plasma and tissue extracts [10-13]. However, interfering of monoterpene isomers remains challenging for the identification and quantification of BP from complex matrix, especially for benzoylalbiflorin (BA), a BP isomer with equivalent groups of benzoic acid and glucose and similar structure of aglycone [14]. In previous research, BA coexisted with BP in the methanolic extract of the roots of Paeonia lactiflora and resembled BP in most of diagnostic fragmentations or characteristic ions on ESI-MS/MS spectra, when LC-MS/MS method was used $[14,15]$. Therefore, new transition pair of the complete removal of BA cross-talking is essential for the selective and robust determination. The investigation of BA will take specific insight into the identification and quantification of BP in rat plasma. In addition, solid-phase 
extraction (SPE) is not suitable for the high-throughput analysis of many plasma samples and is not considered in our experiments due to time consumption and several steps.

In this study, we have developed a rapid and specific LCMS/MS method for the determination of BP in rat plasma, which is simple, time-saving, operational, and distinguished from BA. Additionally, method validation according to Chinese Pharmacopoeia (2010 Edition) and statistical evaluation of data will be conducted. If improved, it may also be applied to other administration routes and other animal species or humans.

\section{Experimental}

2.1. Chemicals. Puerarin (internal standard (IS), $N>98.0 \%$ purity) was purchased from J\&K Scientific Ltd (Beijing, China). Paeonia lactiflora was supplied by Qixin Chinese medicine particles Co. (Hebei, China) and were identified in accordance with each standard stipulated in the Chinese Pharmacopoeia (2010 Edition). BP (99\% purity) and BA (99\% purity) standards were prepared in our laboratory from $\mathrm{Paeo-}$ nia lactiflora. Methanol (HPLC Grade) was purchased from Thermo Fisher Scientific (MA, USA). Ultrapure water was prepared using a Milli-Q Reagent Water System (Millipore, MA, USA) throughout the study.

2.2. Preparation of Standard and Quality Controls. Each stock solution of BP, BA, and IS was prepared at $1 \mathrm{mg} / \mathrm{mL}$ in methanol. Working standard solutions for calibration and controls were prepared by serial dilution of the stock solution with methanol. The working standard solution for IS (100 ng/mL) was prepared by diluting its stock solution with methanol. Calibration standards were prepared by spiking blank rat plasma with appropriate amounts of the working solutions yielding final concentrations of 1, 2, 5, 10, 50, 100, 500 , and $1000 \mathrm{ng} / \mathrm{mL}$ for BP and BA. Quality control (QC) samples for $\mathrm{BP}$ and $\mathrm{BA}$ were prepared by the same way to achieve $1 \mathrm{ng} / \mathrm{mL}, 2 \mathrm{ng} / \mathrm{mL}, 500 \mathrm{ng} / \mathrm{mL}$, and $750 \mathrm{ng} / \mathrm{mL}$. All stock and working solutions were stored prior to validation at $-20^{\circ} \mathrm{C}$ and $4^{\circ} \mathrm{C}$, respectively, and brought to room temperature before use.

2.3. HPLC Conditions. All samples were analyzed by isocratic elution. Chromatographic separation was carried out on Ultimate 3000 with a Hypersil $\mathrm{C}_{18}$ column $(2.1 \times 50 \mathrm{~mm}$, $3 \mu \mathrm{m}$; Thermo Fisher Scientific, USA) at a flow rate of $150 \mu \mathrm{L} / \mathrm{min}$ with the temperature maintained at $30^{\circ} \mathrm{C}$. The mobile phase was acetonitrile/water $(90: 10, \mathrm{v} / \mathrm{v})$ containing $0.1 \%$ formic acid. Each running could be closed in 4 minutes.

2.4. Mass Spectrometry Conditions. The LC system was monitored with triple quadrupole tandem mass spectrometry (API 4000, AB Sciex, CA, USA) equipped with an TurboV electrospray ionization (ESI) source for positive and negative mode. Multiple reaction monitoring (MRM) data were acquired and processed with the Analyst software (AB Sciex, MA, USA). The optimized operation parameters of MRM are listed in Table 1 .
2.5. Method Validation. The selectivity of the method was evaluated by analyzing six lots of blank rat plasma, blank plasma spiked with $\mathrm{BP}, \mathrm{BA}$, and IS, and a rat plasma sample after oral administration of BP. Calibration curves were constructed by analyzing spiked calibration samples on three separate days. Peak area ratios of analyte to IS were plotted against analyte concentrations, and standard curves were well fitted to the equations by linear regression in the concentration range of $1-1000 \mathrm{ng} / \mathrm{mL}$. The lower limit of quantification (LLOQ) is the lowest concentration of analyte which can be quantified reliably, with an acceptable accuracy $(80-120 \%)$ and precision $(<20 \%)$. The LLOQ is considered as the lowest calibration standard.

Precision and accuracy were assessed by the determination of QC samples in six replicates in three validation days. The precision was reflected by relative standard deviation (RSD) and the accuracy by relative error (RE). The intraday and interday RSD value should not exceed $15 \%$ for the QC samples.

The extraction recovery was evaluated by comparing the peak area of extracted QC samples with those of reference QC solutions reconstituted in blank plasma extracts $(n=6)$. To evaluate the matrix effect, blank rat plasma was extracted and then spiked with QC samples. The corresponding peak areas were then compared with those of neat standard solutions at equivalent concentrations, and this peak area ratio is defined as the matrix effect.

Stability of analytes in QC samples was determined by analyzing in triplicate under different conditions: three freeze-thaw cycles were carried out, with samples stored at $-20^{\circ} \mathrm{C}$ for $24 \mathrm{~h}$ and thawed to $20^{\circ} \mathrm{C}$. Short-term stability was assessed by storing samples at $25^{\circ} \mathrm{C}$ for $4 \mathrm{~h}$. The long term stability was accessed by storage at $-20^{\circ} \mathrm{C}$ for 30 days. The results were compared with those obtained with freshly prepared QC samples.

2.6. Pharmacokinetic Study. Twelve male Wistar rats (250-300 g) were obtained from Laboratory Animal Centre of Hebei University (Baoding, China). They were kept in an environmentally controlled breeding room for 3 days before starting the experiments and fed with standard laboratory food and water and fasted overnight before dosing. All rats were divided into two groups randomly. One group was administered with an oral dose of $19 \mathrm{mg} / \mathrm{kg}$ of BP and the other was with the same dose of BA.

Blood samples were collected via the postorbital venous plexus veins into $1.5 \mathrm{~mL}$ heparinized polythene tubes at $5,10,15,20,30,40,60,120,240,360,480$ and $540 \mathrm{~min}$ after intragastric administration. The heparinized blood was immediately centrifuged for $10 \mathrm{~min}$ at $4000 \mathrm{rpm} / \mathrm{min}$ to yield the plasma, stored at $-20^{\circ} \mathrm{C}$ until analysis.

The pharmacokinetic analysis was used to determine concentration-time profiles of $\mathrm{BP}$ and $\mathrm{BA}$ in rat plasma after a single intragastric administration. Data of BP and BA concentrations versus time for each rat were analyzed by the DAS software (version 2.0, Mathematical Pharmacology Professional Committee of China, Shanghai, China). BP or BA plasma concentrations at different times were expressed 
TABLE 1: MS parameters for BP, BA, and IS.

\begin{tabular}{lccccc}
\hline Compound & Charge & Retention time $/ \mathrm{min}^{-1}$ & Parent ion & Daughter ion & $\mathrm{Collision} \mathrm{energy/eV}^{-1}$ \\
\hline IS & Positive & 1.50 & 417.38 & 296.90 & 45 \\
BP & Negative & 2.81 & 583.18 & 165.05 & 25 \\
BA & Positive & 2.91 & 607.18 & 589.10 & 25 \\
\hline
\end{tabular}

TABLE 2: Regression equation and correlation coefficients for BP and BA ( $y=$ peak area ratio of BP or BA versus IS; $x=$ concentration of BP or BA).

\begin{tabular}{lclc}
\hline Compound & Linear range/ng mL & Regression equation & Correlation coefficient \\
\hline BP & $1-1000$ & $y=0.0996 x+0.2805$ & 0.9998 \\
BA & $1-1000$ & $y=0.0985 x-0.2017$ & 0.9982 \\
\hline
\end{tabular}

as mean \pm SD and the concentration-time curve was plotted. The pharmacokinetic parameters of the BP and BA with the $t$ test were analyzed using SPSS 18.0 statistical software. A value of $p<0.05$ was considered statistically significant.

\section{Results and Discussion}

3.1. Chromatographic Condition and MS Parameter Optimization. To avoid residual signals and inaccuracies, the chromatographic conditions were established in isocratic mode for sample analysis. The peak shapes and MS signals of the analytes were improved by using a mobile phase of acetonitrile/water $(90: 10, \mathrm{v} / \mathrm{v})$ containing formic acid $(0.1 \%$, $\mathrm{v} / \mathrm{v})$. Each running could be finished in 4 minute.

Owing to lack knowledge about BA, there are still some imperfections in the appraisal of the BP quantification. To distinguish between $\mathrm{BP}$ and $\mathrm{BA}$, multiple reaction monitoring (MRM) of the transitions were $m / z 583.18 \rightarrow m / z 165.05$ for BP in negative mode, $m / z 607.18 \rightarrow 589.10$ for BA, and $\mathrm{m} / z 417.38 \rightarrow 296.90$ for IS in positive mode, respectively (Figure 1). The collision energy determining product ion signal intensity was $25 \mathrm{eV}$ for both $\mathrm{BP}$ and BA, while $45 \mathrm{eV}$ was used for IS. The optimized MRM parameters are listed in Table 1. During the process of selection on MRM transitions, positive and negative modes were investigated to obtain the optimal transition-pair ions. The specificity of each transition channel was monitored and assessed on BP and BA references. In positive mode, ion $m / z 607$ from BP produced main daughter ions including 485, 411, 375, 341, 289 and 218, while ion $\mathrm{m} / z 607$ from BA produced main daughter ions including $589,485,411,341,289$ and 218 . The structure elucidation was shown in Figure 2 and supplementary information (Figure S1 and Figure S3 in Supplementary Material available online at https://doi.org/10.1155/2017/1693464). Obviously, daughter ions of $m / z 375$ in BP and $m / z 589$ in BA should verify the specificity of $m / z 607 \rightarrow 375$ and $m / z 607 \rightarrow 589$ transitions. In negative mode, ion $m / z 583$ from BP produced main daughter ions including 553,431, 165 and 121, while ion $\mathrm{m} / z$ 583 from BA produced main daughter ions including 431, 195 and 121. The structure elucidation could be seen in Figure 2 and supplementary information (Figure S2 and Figure S4). Daughter ions of $m / z 553,165$ in BP and $m / z 195$ in BA were collected to verify the specificity of $m / z 583 \rightarrow 165$ and $m / z$ $583 \rightarrow 195$ transitions. Specificity experiments indicated that ions on $m / z 583 \rightarrow 553, m / z 583 \rightarrow 195$ in negative mode, and $m / z 607 \rightarrow 375$ in positive mode caused cross-activity on $\mathrm{BP}$ and $\mathrm{BA}$ references, which hints that lower relative abundance of particle could influence the specificity on MRM experiments (supplementary information, Figure S5). However, ions on $\mathrm{m} / \mathrm{z} 583 \rightarrow 165$ in negative mode and $\mathrm{m} / \mathrm{z}$ $607 \rightarrow 589$ in positive mode were specific for BP and BA, respectively (Figure 3 ). Therefore, the above two channels could be used to the following studies.

3.2. Method Validation. The MRM chromatogram of blank plasma, blank plasma spiked with BP, BA, and IS, and samples collected 20 min after intragastric administration with BP are displayed in Figure 4. Although the different retention time between BP and BA is only $0.1 \mathrm{~min}$, excellent peak shapes were exhibited for both analytes and IS, indicating no significant interference from endogenous substances near BP, $\mathrm{BA}$, or IS retention times.

Peak area ratios of BP or BA to IS $(y)$ versus analyte concentrations $(x)$ were fitted at the concentration range of $1-1000 \mathrm{ng} / \mathrm{mL}$ in rats plasma. Typical equations for the calibration curves are listed in Table 2 . The correlation coefficients for BP and BA were 0.9998 and 0.9982 (>0.995), with the equations showing good linearity between peak areas ratios and concentrations. Under optimized conditions, The LLOQ for $\mathrm{BP}$ and $\mathrm{BA}$ were 1.0 and $1.0 \mathrm{ng} / \mathrm{mL}$, respectively.

The precision and accuracy of intra- and interday measurements were evaluated using QC sample. The data obtained for BP and BA $(n=6)$ are summarized in Table 3. Interday precision and accuracy were determined on five consecutive days. The intraday and interday precisions of $\mathrm{BP}$ and $\mathrm{BA}$ ranged from 2.03 to $12.48 \%$. The accuracies of $\mathrm{BP}$ and $\mathrm{BA}$ ranged from -8.00 to $10.33 \%$. These findings indicated that precision and accuracy were within the acceptable limits.

The mean recovery rates for BP and BA were from $93 \pm$ $14.29 \%$ to $99 \pm 6.62 \%$ (Table 3 ). The data demonstrated that the extraction method was consistent and reproducible at different QC levels and suitable for BP and BA. The matrix effects for BP and BA were within $93 \pm 14.29 \%$ to $99 \pm 14.29 \%$. Ion suppression caused by the plasma matrix was negligible. 


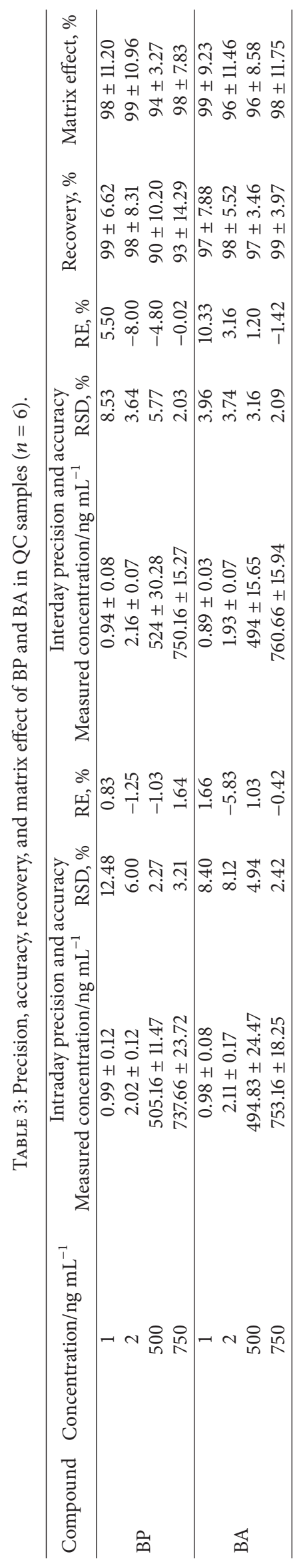




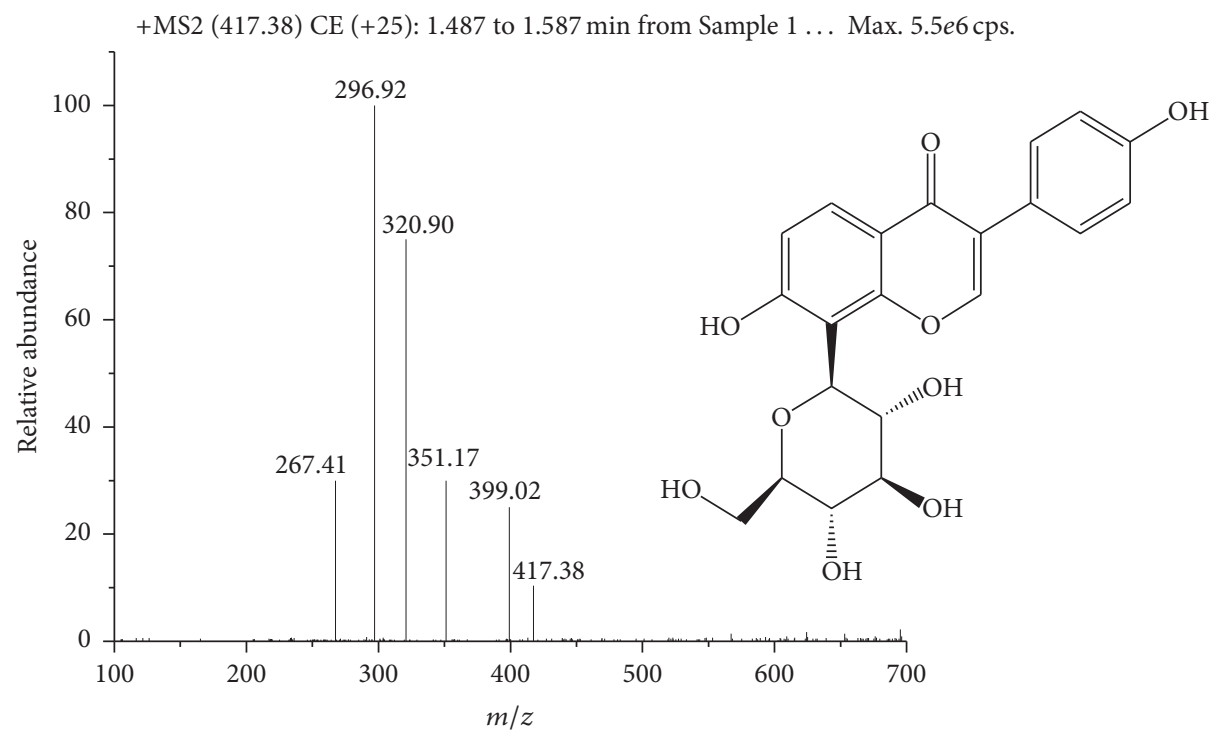

(a)

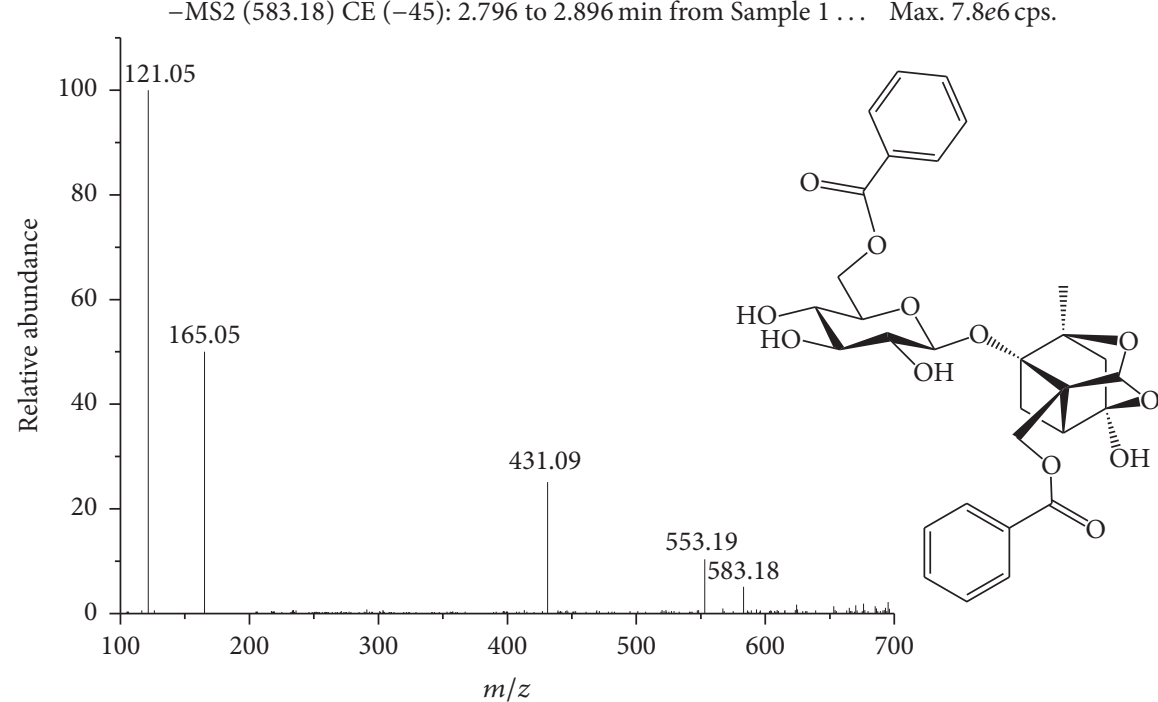

(b)

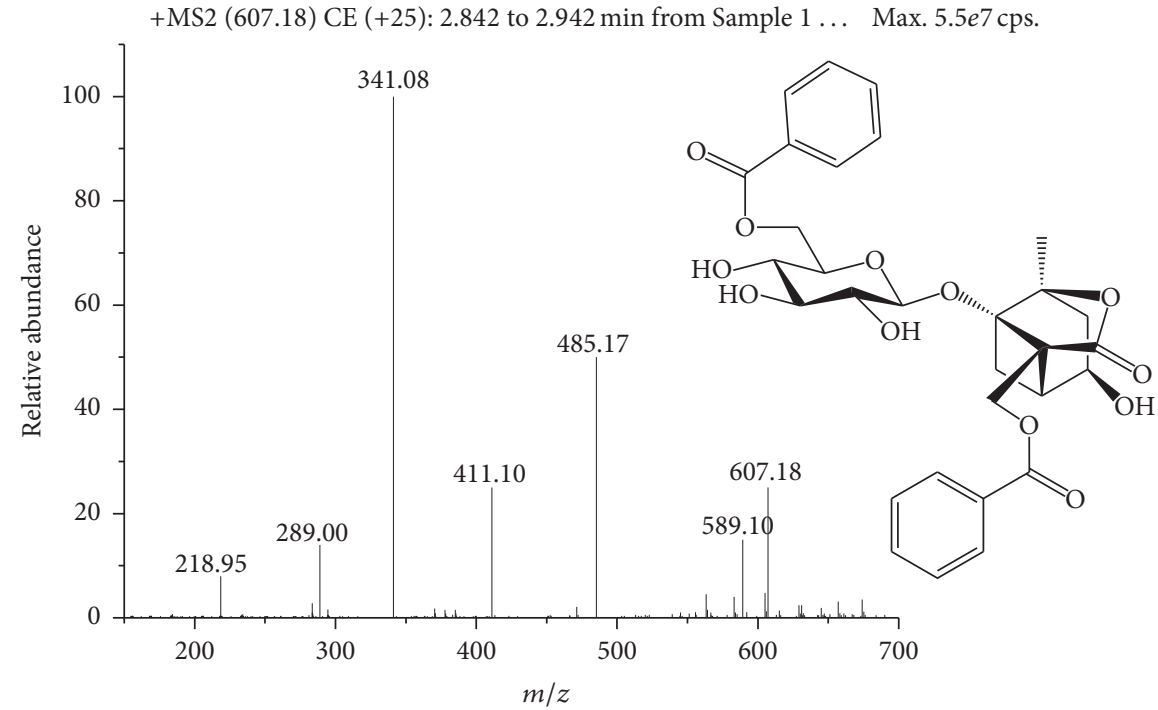

(c)

FIGURE 1: MS/MS spectra and structures of the two analytes and IS: (a) IS, parent ion: $m / z$ 417.38; daughter ion: $m / z$ 296.90; (b) BP, parent ion: $m / z$ 583.18; daughter ion: $m / z$ 165.05; (c) BA, parent ion: $m / z$ 607.18; daughter ion: $m / z 589.10$. 
$\mathrm{BP}$

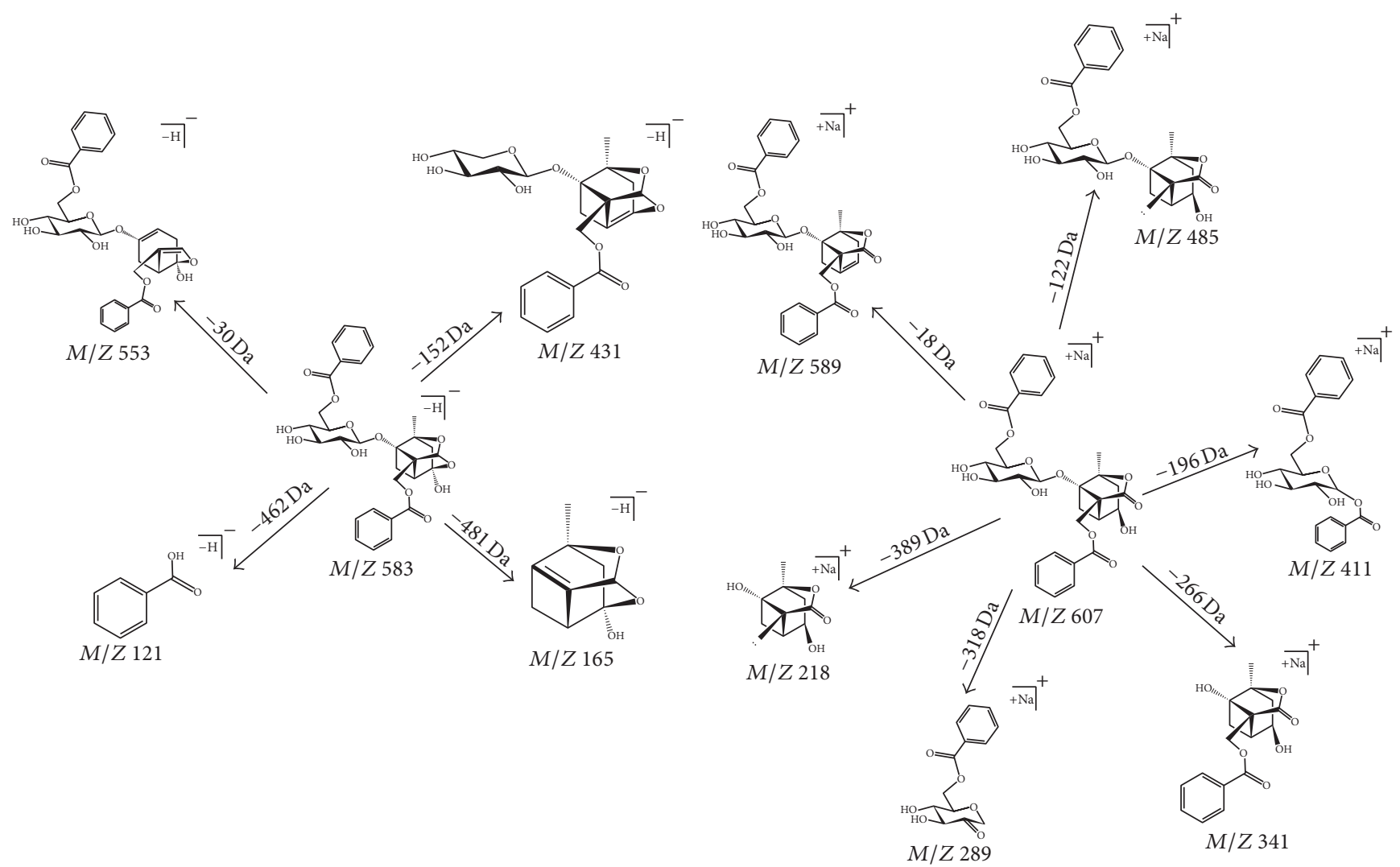

FIGURE 2: The structure elucidation on BP in negative mode and BA in positive mode.

TABLE 4: BP and BA stability in QC samples $(n=6)$.

\begin{tabular}{ccccccccccc}
\hline \multirow{2}{*}{ Compound Concentration/ng mL } & \multicolumn{3}{c}{ Three freeze thaw cycles } & \multicolumn{3}{c}{ Short-term for $4 \mathrm{~h}\left(25^{\circ} \mathrm{C}\right)$} & \multicolumn{3}{c}{ Long-term for 30 Days $\left(25^{\circ} \mathrm{C}\right)$} \\
& & Mean $\pm \mathrm{SD}$ & $\mathrm{RSD}, \%$ & $\mathrm{RE}, \%$ & Mean $\pm \mathrm{SD}$ & $\mathrm{RSD}, \%$ & $\mathrm{RE}, \%$ & $\mathrm{Mean} \pm \mathrm{SD}$ & $\mathrm{RSD}, \%$ & $\mathrm{RE}, \%$ \\
\hline & 1 & $0.99 \pm 0.03$ & 3.77 & 0.16 & $0.91 \pm 0.05$ & 5.60 & 9.00 & $0.88 \pm 0.09$ & 10.99 & 11.50 \\
$\mathrm{BP}$ & $2.04 \pm 0.18$ & 9.14 & -2.00 & $1.85 \pm 0.07$ & 3.91 & 7.33 & $1.81 \pm 0.08$ & 4.79 & 9.50 \\
& 400 & $382.33 \pm 32.20$ & 8.42 & 4.41 & $397.50 \pm 9.18$ & 2.30 & 0.62 & $382.66 \pm 11.86$ & 3.09 & 4.33 \\
& 750 & $747.50 \pm 9.87$ & 1.32 & 0.33 & $743.00 \pm 15.15$ & 2.03 & 0.93 & $740.50 \pm 4.18$ & 0.56 & 1.26 \\
\hline \multirow{3}{*}{$\mathrm{BA}$} & $0.94 \pm 0.06$ & 6.38 & 6.00 & $0.93 \pm 0.03$ & 3.90 & 7.00 & $0.91 \pm 0.04$ & 4.86 & 9.00 \\
& 1 & $1.96 \pm 0.10$ & 5.27 & 1.91 & $2.00 \pm 0.07$ & 3.69 & -0.41 & $2.19 \pm 0.18$ & 8.24 & -9.83 \\
& 4 & $404 \pm 8.09$ & 2.00 & -1.00 & $397.66 \pm 16.04$ & 4.03 & 0.58 & $396.83 \pm 16.19$ & 4.08 & 0.79 \\
& 700 & $746 \pm 5.17$ & 0.69 & 0.53 & $755.83 \pm 13.99$ & 1.85 & -0.77 & $757.66 \pm 17.71$ & 2.33 & -1.02 \\
\hline
\end{tabular}

Stability was determined by analyzing QC samples in triplicate and three different conditions. The results are displayed in Table 4. BP, BA, and IS were stable in plasma at room temperature for $4 \mathrm{~h}$, after three freeze-thaw cycles, and at $-20^{\circ} \mathrm{C}$ for at least one month. These results indicated the method was accurate, reliable, and reproducible.

3.3. Pharmacokinetic Study. To further assess the pharmacokinetics of BP in rats, the main pharmacokinetic parameters of BP and BA were compared in Table 5 using a noncompartmental model (Figure 5). $T_{\max }$ and $t_{1 / 2}$ were $0.35 \pm 0.07$ and $1.97 \pm 0.23$ for $\mathrm{BP}$ and $0.31 \pm 0.03$ and $1.47 \pm 0.30$ for BA which showed no significant differences between BP and BA. However, $C_{\max }, \mathrm{AUC}_{0-t}$, and $\mathrm{MRT}_{0-\infty}$ were slightly higher in $\mathrm{BP}$ than in $\mathrm{BA}$. To investigate pharmacokinetic profiles of $\mathrm{BP}$ and $\mathrm{BA}$, these findings indicated that further researches need focus on the distribution of $\mathrm{BP}$ and $\mathrm{BA}$ in different tissues. 
TABLE 5: Pharmacokinetic parameters of BP and BA in rats after intragastric administration.

\begin{tabular}{|c|c|c|c|}
\hline \multirow{2}{*}{ Parameters } & \multirow{2}{*}{ Unit } & \multicolumn{2}{|c|}{ Values } \\
\hline & & BP & BA \\
\hline$t_{1 / 2}{ }^{*}$ & $\mathrm{~h}$ & $1.97 \pm 0.23$ & $1.47 \pm 0.30$ \\
\hline AUC $(0-\infty)^{*}$ & $\mathrm{hng} \mathrm{mL^{-1 }}$ & $102.87 \pm 31.74$ & $65.81 \pm 15.24$ \\
\hline $\operatorname{MRT}(0-\infty)^{*}$ & $\mathrm{~h}$ & $2.27 \pm 0.32$ & $2.01 \pm 0.15$ \\
\hline$T_{\max }{ }^{*}$ & $\mathrm{~h}$ & $0.35 \pm 0.07$ & $0.31 \pm 0.03$ \\
\hline $\mathrm{Cl}^{*}$ & $\mathrm{~h}$ & $1.15 \pm 0.34$ & $1.74 \pm 0.36$ \\
\hline$V_{d}{ }^{*}$ & $\mathrm{~L} / \mathrm{h} / \mathrm{kg}$ & $3.71 \pm 0.35$ & $3.79 \pm 1.47$ \\
\hline$C_{\max }^{*}$ & $\mathrm{ng} / \mathrm{mL}$ & $57.23 \pm 17.18$ & $49.76 \pm 3.51$ \\
\hline
\end{tabular}

BP reference
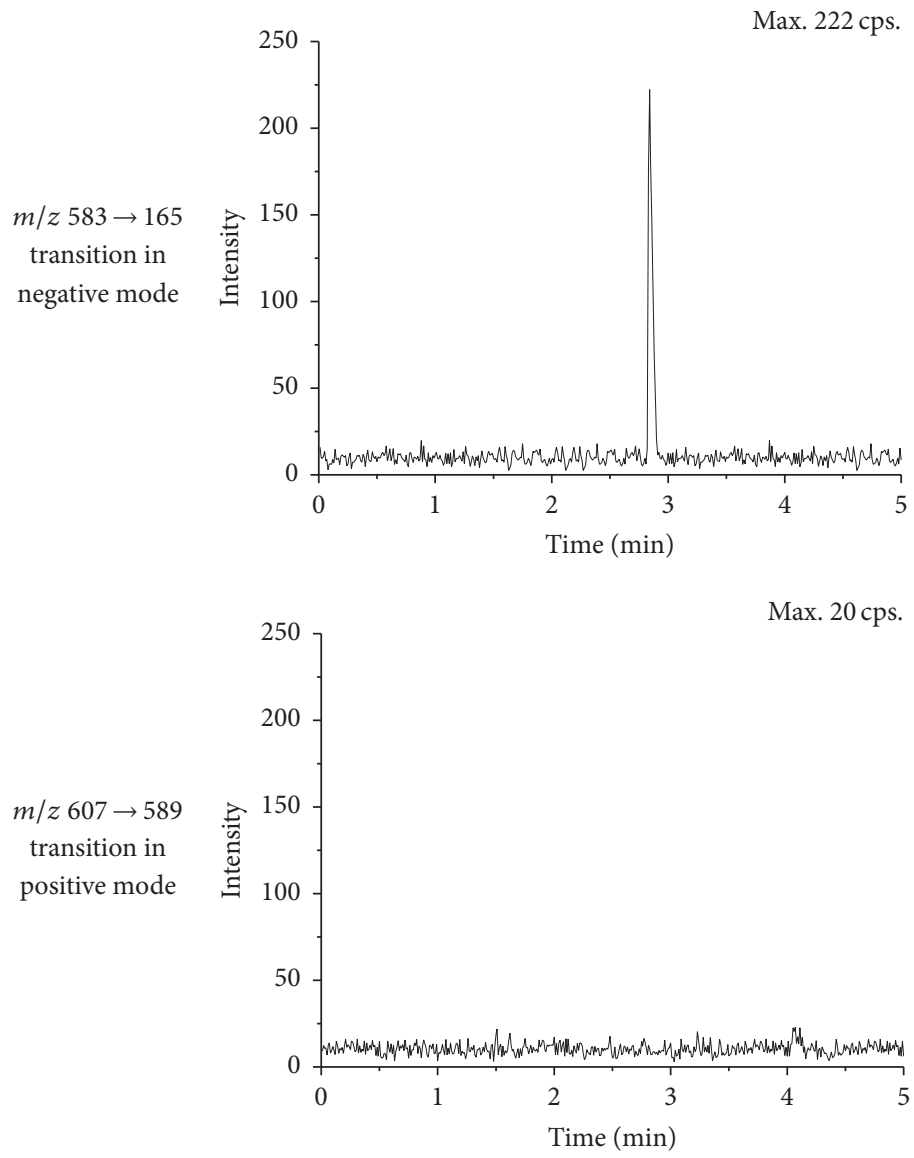

BA reference
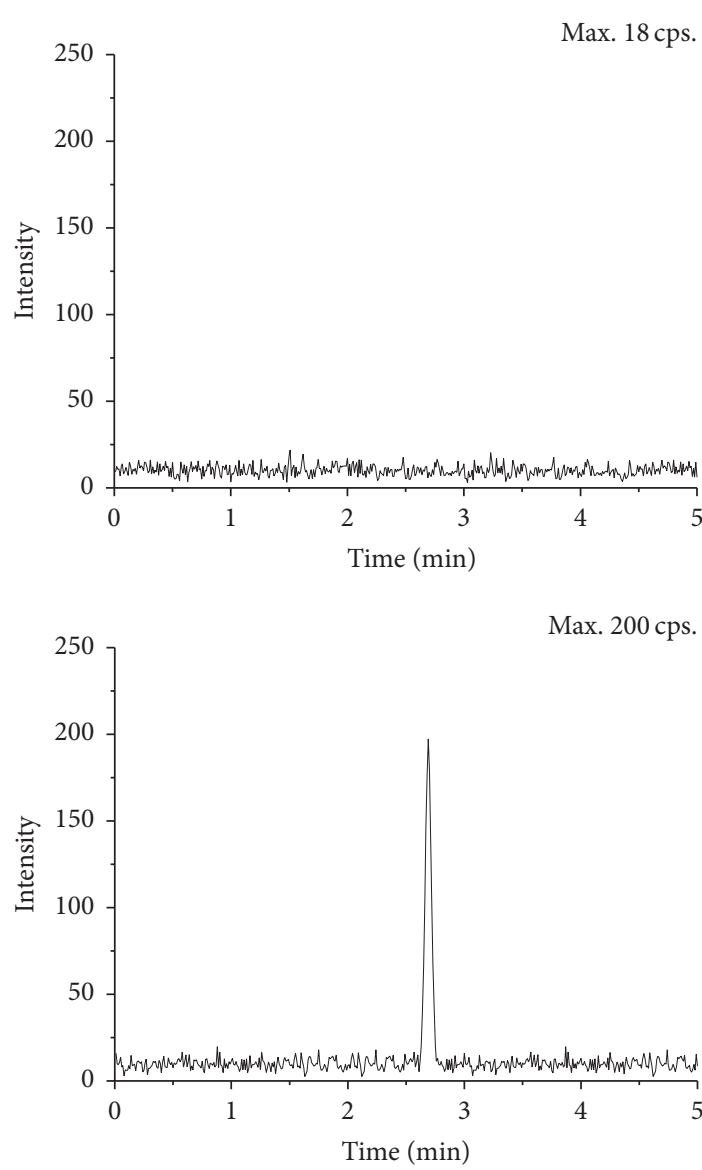

FIGURE 3: The specificity of $\mathrm{m} / z 583 \rightarrow 165$ in negative mode and $\mathrm{m} / z 607 \rightarrow 589$ positive mode on BP and BA references.

\section{Conclusion}

This study is the first validated biological analysis of BP in rat plasma distinguishing with BA isomer. This proposed method was successfully applied to pharmacokinetic study of $\mathrm{BP}$ and $\mathrm{BA}$, which presented the advantages of high specificity, sensitivity, relatively simple preparation, and high extraction recovery rate. Similar pharmacokinetic curves and parameters were obtained for $\mathrm{BP}$ and $\mathrm{BA}$, excluding $C_{\max }$ and AUC parameters. The pharmacokinetic study showed that $\mathrm{BP}$ and $\mathrm{BA}$ were quickly eliminated in rats. Therefore, both BP and BA, likely, could play an important role in the pharmacological effects.

\section{Conflicts of Interest}

The authors declare that they have no conflicts of interest. 

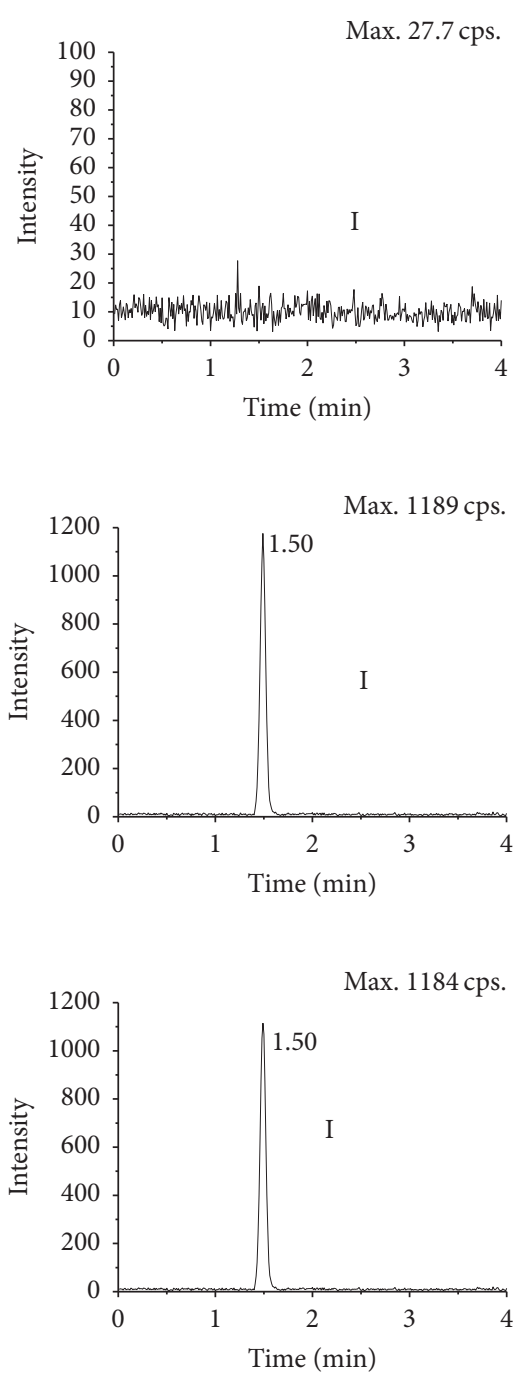

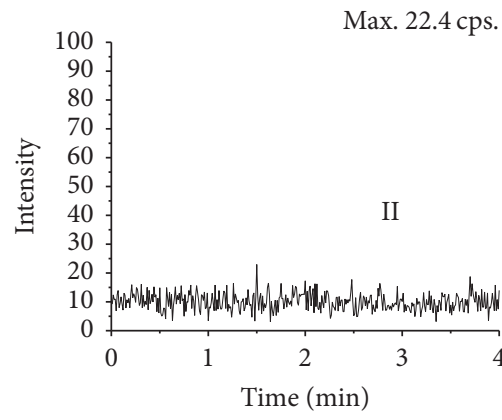

(a)

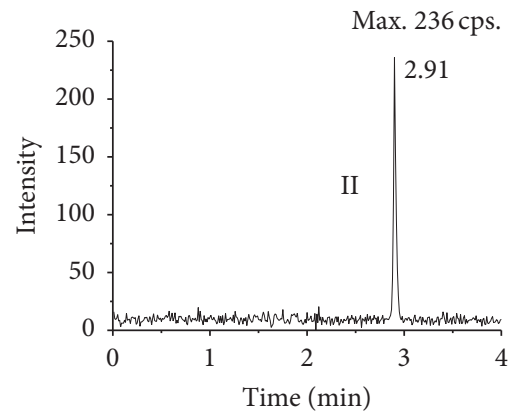

(b)

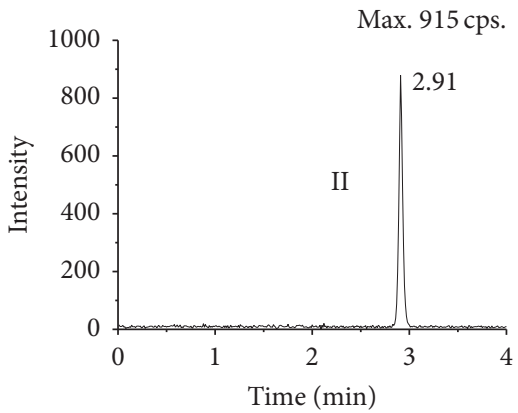

(c)
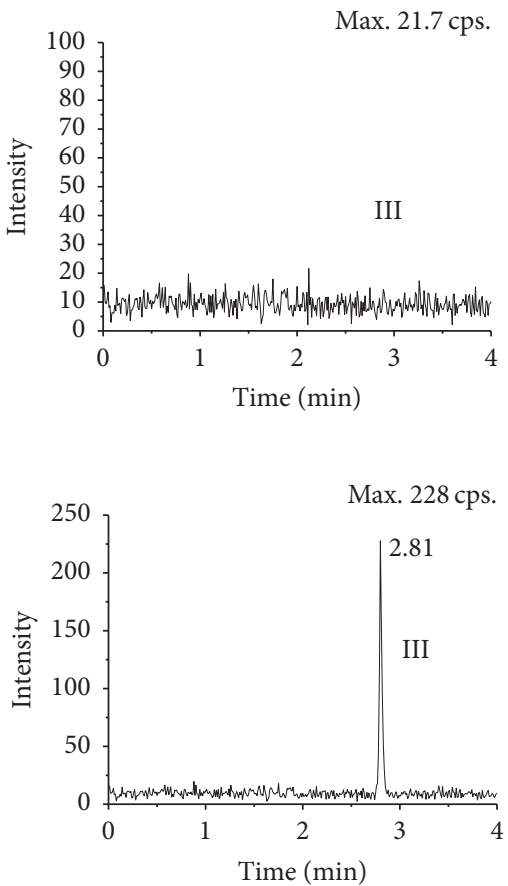

Max. $17.5 \mathrm{cps}$.

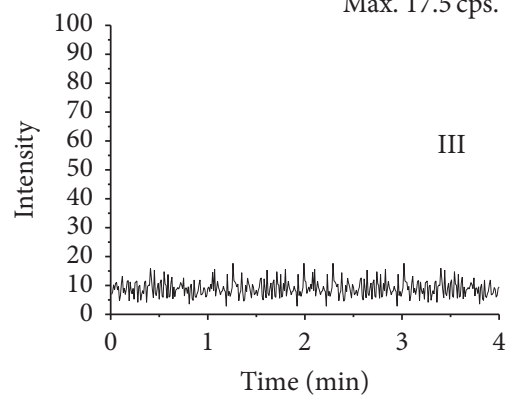

FIgURE 4: Typical MRM chromatograms of IS, BP, and BA: (a) blank rat plasma; (b) blank rat plasma spiked with IS (I, $100 \mathrm{ng} / \mathrm{mL}$ ), BP $(1 \mathrm{ng} / \mathrm{mL})$, and BA $(1 \mathrm{ng} / \mathrm{mL})$; (c) rat plasma sample at $20 \mathrm{~min}$ after intragastric administration of BP spiked with IS; Roman numerals: I for IS, II for BP, and III for BA.

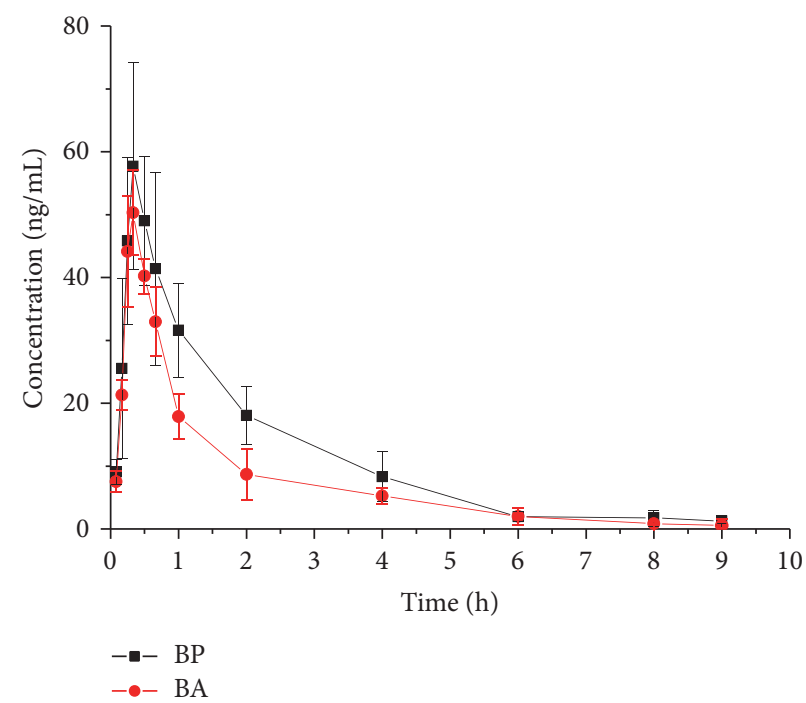

FIGURE 5: Mean plasma concentration-time profiles of BP and BA in rats after intragastric administration. Data are mean \pm SD $(n=6)$. 


\section{Acknowledgments}

This study was supported by the Natural Science Foundation of Hebei Province (China, no. B2012201009) and Natural Science Foundation of Hebei University (China, no. 33331112).

\section{References}

[1] A. Burns and S. Iliffe, "Alzheimer's disease," BMJ, vol. 338, article b158, 2009.

[2] World Health Organization, 2015, http://www.who.int/mediacentre/factsheets/fs362/en/.

[3] B. Halliwell, J. M. C. Gutteridge, and C. E. Cross, "Free radicals, antioxidants, and human disease: where are we now?" The Journal of Laboratory and Clinical Medicine, vol. 119, no. 6, pp. 598-620, 1992.

[4] W. R. Markesbery, "The role of oxidative stress in Alzheimer disease," Archives of Neurology, vol. 56, no. 12, pp. 1449-1452, 1999.

[5] S. H. Kim, M. K. Lee, K. Y. Lee, S. H. Sung, J. Kim, and Y. C. Kim, "Chemical constituents isolated from Paeonia lactiflora roots and their neuroprotective activity against oxidative stress in vitro," Journal of Enzyme Inhibition and Medicinal Chemistry, vol. 24, no. 5, pp. 1138-1140, 2009.

[6] M. Kong, H.-H. Liu, J. Xu et al., "Quantitative evaluation of Radix Paeoniae Alba sulfur-fumigated with different durations and purchased from herbal markets: simultaneous determination of twelve components belonging to three chemical types by improved high performance liquid chromatographydiode array detector," Journal of Pharmaceutical and Biomedical Analysis, vol. 98, pp. 424-433, 2014.

[7] X. Zhu and Z.-H. Fang, "New monoterpene glycosides from the root cortex of Paeonia suffruticosa and their potential antiinflammatory activity," Natural Product Research, vol. 28, no. 5, pp. 301-305, 2014.

[8] Y. Cheng, C. Peng, F. Wen, and H. Zhang, "Pharmacokinetic comparisons of typical constituents in white peony root and sulfur fumigated white peony root after oral administration to mice," Journal of Ethnopharmacology, vol. 129, no. 2, pp. 167-173, 2010.

[9] J.-H. Kim, C.-S. Seo, and H.-K. Shin, "Development of validated determination of the eleven marker compounds in Gyejibokryeong-hwan for the quality assessment using HPLC analysis," Archives of Pharmacal Research, vol. 38, no. 1, pp. 5262, 2015.

[10] D. Wu, J. Chen, H. Zhu et al., "UPLC-PDA determination of paeoniflorin in rat plasma following the oral administration of Radix Paeoniae Alba and its effects on rats with collageninduced arthritis," Experimental and Therapeutic Medicine, vol. 7, no. 1, pp. 209-217, 2014.

[11] X. Li, F. Shi, P. Gu, L. Liu, H. He, and L. Ding, "A sensitive LCMS/MS method for simultaneous determination of amygdalin and paeoniflorin in human plasma and its application," Journal of Pharmaceutical and Biomedical Analysis, vol. 92, pp. 160-164, 2014.

[12] X. Huang, S. Su, W. Cui et al., "Simultaneous determination of paeoniflorin, albiflorin, ferulic acid, tetrahydropalmatine, protopine, typhaneoside, senkyunolide I in Beagle dogs plasma by UPLC-MS/MS and its application to a pharmacokinetic study after Oral Administration of Shaofu Zhuyu Decoction," Journal of Chromatography B: Analytical Technologies in the Biomedical and Life Sciences, vol. 962, pp. 75-81, 2014.
[13] J. Ma, S. Wang, M. Zhang et al., "Simultaneous determination of bupropion, metroprolol, midazolam, phenacetin, omeprazole and tolbutamide in rat plasma by UPLC-MS/MS and its application to cytochrome P450 activity study in rats," Biomedical Chromatography, vol. 29, no. 8, pp. 1203-1212, 2015.

[14] P. H. Yen, P. van Kiem, N. X. Nhiem et al., "A new monoterpene glycoside from the roots of Paeonia lactiflora increases the differentiation of osteoblastic MC3T3-E1 cells," Archives of Pharmacal Research, vol. 30, no. 10, pp. 1179-1185, 2007.

[15] R. Li, X. Wang, Y. Zhou, M. Cai, and L. Ding, "Analysis of sodium adduct paeoniflorin, albiflorin and their derivatives by $(+)$ ESI-MSn, DFT calculations and computer-aided mass spectrometry analysis program," Journal of Mass Spectrometry, vol. 42, no. 3, pp. 335-345, 2007. 

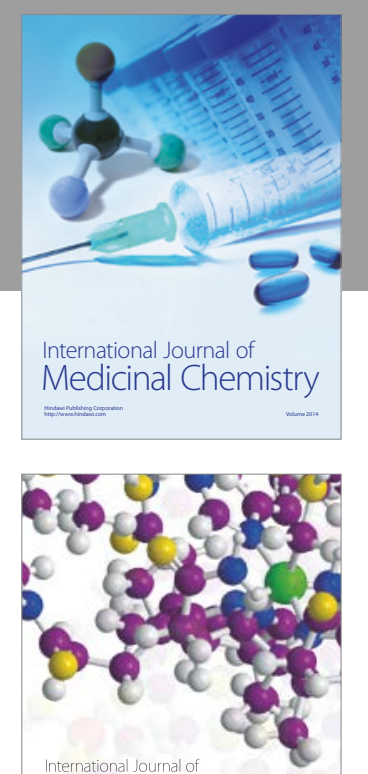

Carbohydrate Chemistry

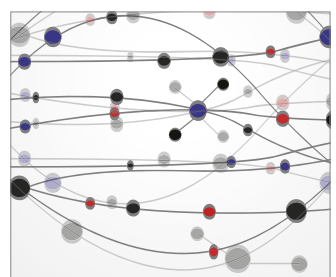

The Scientific World Journal
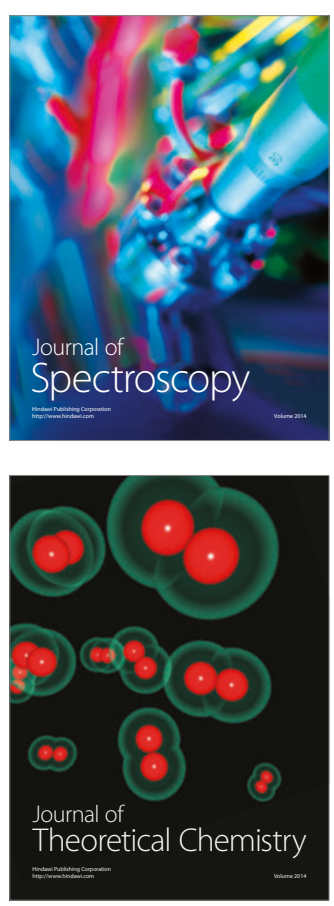
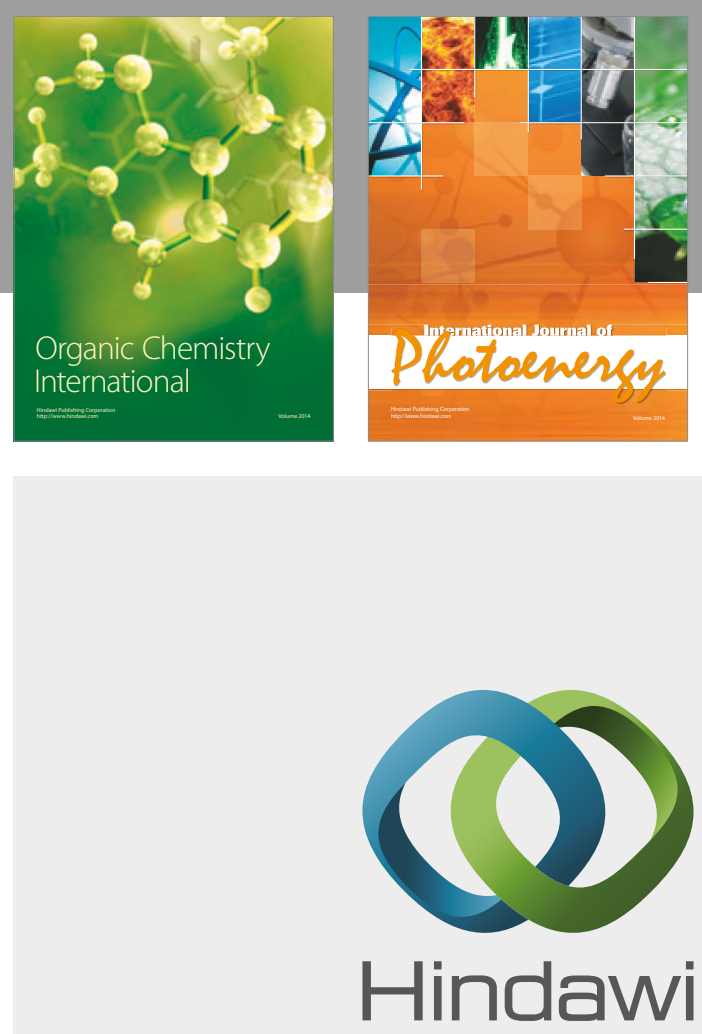

Submit your manuscripts at

https://www.hindawi.com

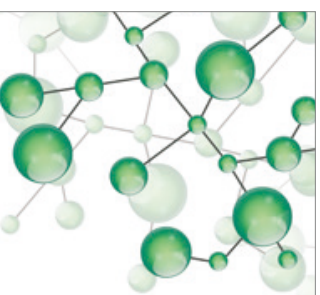

International Journal of

Inorganic Chemistry

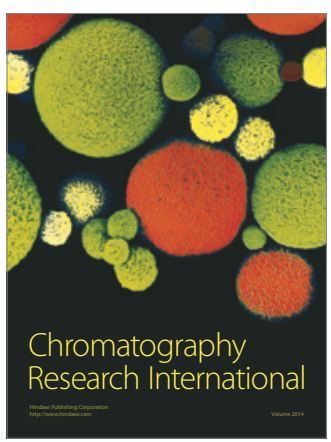

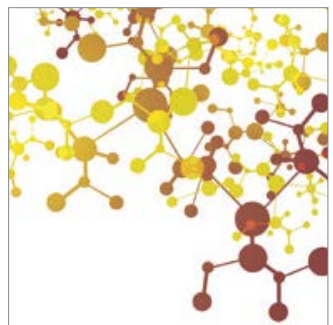

Applied Chemistry
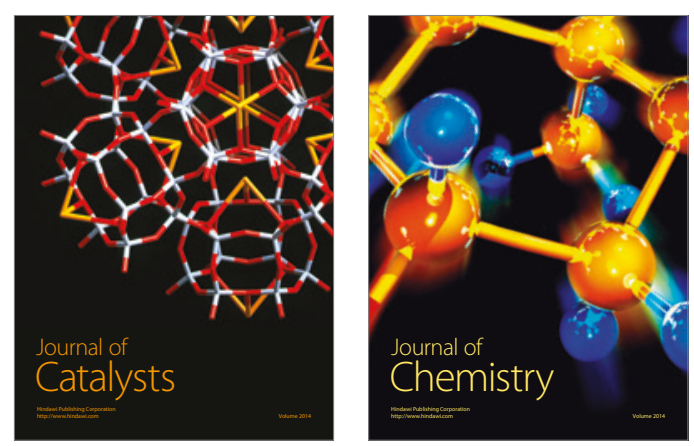
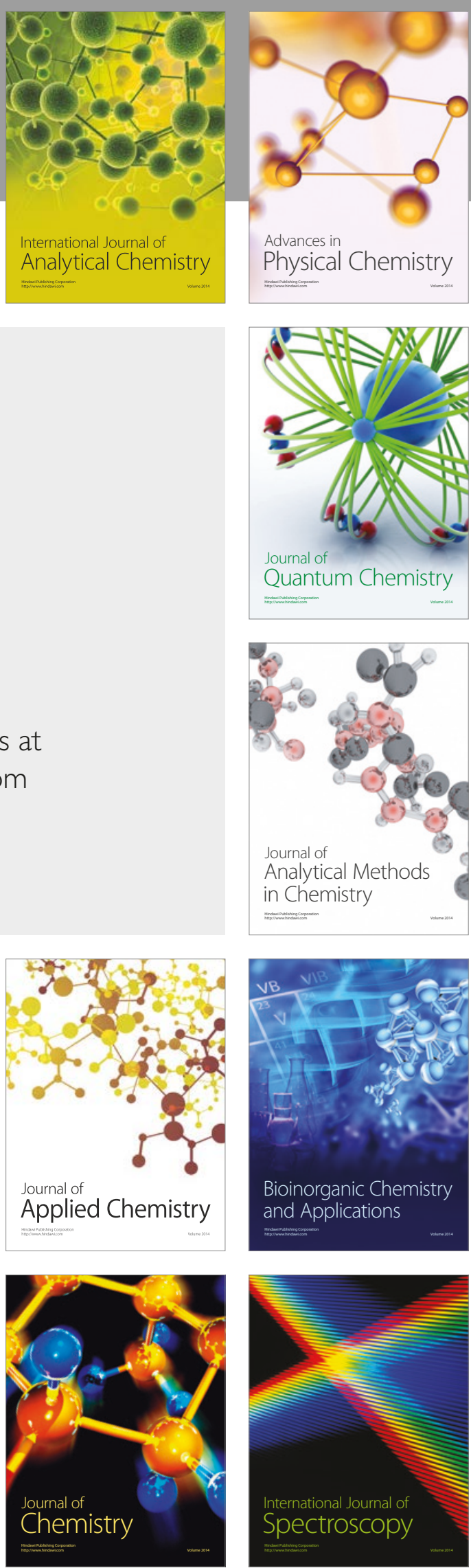